Socioeconomic Institute

Sozialökonomisches Institut

Working Paper No. 0705

Risk and Rationality: Uncovering Heterogeneity in Probability Distortion

Adrian Bruhin, Helga Fehr-Duda, and Thomas F. Epper

July 2007, revised version 
Socioeconomic Institute

University of Zurich

Working Paper No. 0705

\section{Risk and Rationality: Uncovering Heterogeneity in Probability Distortion}

July 2007, revised version

Authors' addresses: $\quad$ Adrian Bruhin

E-mail: adrian.bruhin@sts.uzh.ch

Helga Fehr-Duda

E-mail: fehr@econ.gess.ethz.ch

Thomas F. Epper

E-mail: epper@econ.gess.ethz.ch

Publisher Sozialökonomisches Institut

Bibliothek (Working Paper)

Rämistrasse 71

CH-8006 Zürich

Phone: +41-44-634 2137

Fax: +41-44-634 4982

URL: www.soi.uzh.ch

E-mail: soilib@soi.uzh.ch 


\title{
Risk and Rationality:
}

\section{Uncovering Heterogeneity in Probability Distortion *}

\author{
ADRIAN BRUHIN \\ HELGa FeHR-DudA \\ ThOMAs F. EPPER
}

July 18, 2007

\begin{abstract}
It has long been recognized that there is considerable heterogeneity in individual risk taking behavior but little is known about the distribution of risk taking types. We present a parsimonious characterization of risk taking behavior by estimating a finite mixture regression model for three different experimental data sets, two Swiss and one Chinese, over a large number of real gains and losses. We find two distinct types of individuals: In all three data sets, the choices of roughly $80 \%$ of the subjects exhibit significant deviations from rational probability weighting consistent with prospect theory. $20 \%$ of the subjects weight probabilities linearly and behave essentially as expected value maximizers. Moreover, the individuals are assigned to one of these two groups with probabilities of close to one resulting in a low measure of entropy. The reliability and robustness of our classification suggest using a mix of preference theories in applied economic modeling.
\end{abstract}

KEYWORDS: Individual Risk Taking Behavior, Latent Heterogeneity, Finite Mixture Regression Models

JEL CLASSIFICATION: D81, C49

*Authors' affiliation: Swiss Federal Institute of Technology, Chair of Economics, Weinbergstrasse 35, CH8092 Zurich, Switzerland, phone: +41 44632 4625, email of corresponding author: adrian.bruhin@sts.uzh.ch 


\section{Introduction}

Risk is a ubiquitous feature of social and economic life. Many of our everyday choices, and often the most important ones, such as what trade to learn and where to live, involve risky consequences. While it has long been recognized that individuals differ in their risk taking attitudes, surprisingly little is known about the distribution of risk preferences in the population (for an exception see Dohmen, Falk, Huffman, Sunde, Schupp, and Wagner (2005)). Since preferences are one of the ultimate drivers of behavior, knowledge of the composition of risk attitudes is paramount to predicting economic behavior. Economic models often allow for heterogeneity, but this heterogeneity is usually confined to remain within the boundaries of the standard model of preferences, expected utility theory (EUT). The empirical evidence, however, reveals that heterogeneity in risk taking behavior is of a substantive kind, i.e. some people evaluate risky prospects consistently with EUT, whereas other people deviate substantially from expected utility maximization (Hey and Orme, 1994). Moreover, it seems to be the case that rational decision makers revealing EUT-preferences constitute only a minority of the population. To improve descriptive performance a plethora of alternative theories have been developed (for an overview see Starmer (2000)). Unfortunately, no single best fitting model has been identified so far (Harless and Camerer, 1994) and, depending on the individual, one or the other model fits better. This finding poses a serious problem for applied economics. What the modeler needs is a parsimonious representation of risk preferences which is empirically well grounded and robust, and not a host of different functionals. Providing such a parsimonious characterization of heterogeneity in risk taking behavior is the objective of this paper.

Our method is based on a literature on classifying individuals which has recently emerged in the social sciences. On the basis of statistical classification procedures, such as finite mixture regression models, investigators have tried to discover which decision rules people actually use when playing games or dealing with complex decision situations (El-Gamal and Grether, 1995; Houser, Keane, and McCabe, 2004). The finite mixture regression approach does not require fitting a model for each individual which is - given the usual quality of the choice data 
- frequently impossible. Instead, our approach reveals latent heterogeneity by estimating the relative sizes of distinct behavioral groups and by endogenously assigning each individual to a specific group characterized by a unique set of parameter values.

We apply such a finite mixture regression model to choice data from three different experiments, two of which were conducted in Zurich, Switzerland. The third experiment took place in Beijing, People's Republic of China. We analyze 452 subjects' decisions over real monetary gains and losses, which comprise a total of about 18,000 choices. All three experiments were designed in a similar manner and served to elicit certainty equivalents for binary lotteries. Using a flexible sign-dependent functional as basic behavioral model, we show the following results.

First, in all three data sets, we find two distinct behavioral types of risk taking behavior. Second, the ratios of the different types in their respective populations are practically equal in both the Swiss and the Chinese data sets and amount to roughly 20:80. Third, without putting any a priori restrictions on parameter values we find that one of the two types, which comprises about $20 \%$ of the individuals in each data set, exhibits near linear probability weighting functions and value functions. Therefore, this group can essentially be characterized as expected value maximizers. This result is particularly interesting in the light of Rabin's calibration theorem (Rabin, 2000) which shows that expected utility maximizers should be approximately risk neutral for small stakes typically encountered in laboratory experiments. Therefore, we label subjects belonging to this group of risk neutral people as "EUT-types".

Fourth, in each data set, the second group, which comprises about $80 \%$ of the individuals, is characterized by significant deviations from linear probability weighting and can be conveniently described as prospect theory types. Fifth, almost all the experimental subjects are unambiguously assigned to one of the two distinct types. Measuring the quality of classification by the average normalized entropy (El-Gamal and Grether, 1995) we obtain an extent of ambiguous assignments of less than $5 \%$ of the maximum entropy, a value which is, to our knowledge, unequaled in the literature. Thus, we observe almost no "ambiguous" types, i.e. individuals that are assigned a high probability (of say 0.4) of being one type and a high probability (of say 0.6 ) of being another type are practically absent. This clean classifica- 
tion suggests that the procedure is able to capture an essential manifestation of individual heterogeneity.

Finally, for all three prospect theory groups we obtain similar parameter values for the probability weighting function and the value function over losses, indicating a considerable cross-cultural stability of preference parameters. For decisions over gains, however, Chinese behavior differs substantially from Swiss behavior. Overweighting of probabilities is more pronounced and the sensitivity to changes in probabilities is considerably lower for Chinese subjects. Moreover, the Chinese value function is clearly concave, whereas the corresponding Swiss ones are close to linearity. The total effect of both components render the Chinese relatively more risk seeking for gains over a considerable range of probabilities. Thus, the finite mixture regression helps to better understand the nature of cross-cultural differences.

These results show that the classification procedure successfully uncovers latent heterogeneity in the population. If there is heterogeneity of a substantive kind, as the data suggest, basing predictions on a single preference theory is inappropriate and may lead to biased results. EUT preferences should be taken account of alongside prospect theory preferences even if rational behavior constitutes only a minority in the population. As the literature on the role of bounded rationality under strategic complementarity and substitutability has shown (Haltiwanger and Waldman, 1985, 1989; Fehr and Tyran, 2005; Camerer and Fehr, 2006), the mix of rational and irrational actors may be decisive for aggregate outcomes. Depending on the nature of the strategic interdependence even a minority of players of a particular type may determine the aggregate outcome. Therefore, the mix of types in the population is a crucial variable in predicting market outcomes. Since the finite mixture regression provides a robust and reliable classification of the individuals estimates of group sizes and group-specific parameters may serve as a valuable inputs for applied economics.

To the best of our knowledge, there is no study showing a nearly identical classification of risk preference types for three independent data sets. Related work by Harrison and Rutstroem (2006) and Harrison, Humphrey, and Verschoor (2005) also applies a finite mixture regression model to several experimental data sets but decisively distinguishes itself from our analysis. Their estimation procedure classifies choices assuming one type to be expected util- 
ity maximizing. In contrast, our estimation procedure assigns subjects endogenously to one of two distinct types, one of which turns out to be consistent with EUT-preferences. Thus, our results can be viewed as stronger evidence in favor of a non-negligible share of EUT individuals. In addition, our results show one feature which renders our classification particularly convincing: Almost all individuals are assigned with a probability close to one to one of the two endogenously emerging preference types.

The paper is structured as follows. Section 2 describes the experimental design for the three experiments. The functional specification of the behavioral model and the finite mixture regression model are discussed in Section 3. Section 4 presents descriptive statistics of the data and the results of the classification procedure. Section 5 concludes.

\section{Experimental Design}

In the following section we describe the experimental setup and procedures. The experiments took place in Zurich in 2003 and 2006 as well as in Beijing in 2005. In Zurich, all subjects were recruited from the subject pool of the Institute of Empirical Research in Economics which contains students of all fields of the University of Zurich and the Swiss Federal Institute of Technology Zurich. In Beijing, subjects were recruited by flyer distributed at the campus of Peking University. Since all three experiments are based on the same design principles, we will present the prototype experiment Zurich 2003 in detail (Fehr-Duda, de Gennaro, and Schubert, 2006) and describe to what extent the other two experiments deviate from the prototype. The main distinguishing features of the different experiments are summarized in Table 1.

We elicited certainty equivalents for a large number of two-outcome lotteries. One half of the lotteries was framed as choices between risky and certain gains ("gain domain"). The remaining decisions were presented as choices between risky and certain losses ("loss domain"). For each lottery in the loss domain, subjects were endowed with a specific cash amount which served to cover their potential losses and which rendered the expected payoff for the loss lottery equal to the expected payoff of an equivalent gain lottery. In the Zurich 2003 and the Beijing 
Table 1: Differences in Experimental Design

\begin{tabular}{lccc}
\hline \hline & Zurich 03 & Zurich 06 & Beijing 05 \\
\hline Number of: & & & \\
Subjects & 181 & 118 & 153 \\
Lotteries & 50 & 40 & 28 \\
Observations & 9,005 & 4,669 & 4,281 \\
& & & \\
Procedure & computerized & computerized & paper and pencil \\
Framing & abstract and & contextual & $\begin{array}{c}\text { abstract and } \\
\text { contextual }\end{array}$ \\
& contextual & & \\
\hline \hline
\end{tabular}

experiments, $50 \%$ of the subjects were confronted with decisions framed in the standard gamble format, the other $50 \%$ of the subjects had to make choices framed in contextual terms, i.e. gains were represented as risky or sure investment gains, losses as repair costs and insurance premiums, respectively ${ }^{1}$. The Zurich 2006 experiment was based on contextual lotteries only. Outcomes $x_{1}$ and $x_{2}$ ranged from zero Swiss Francs to 150 Swiss Francs ${ }^{2}$. The payoffs in the Beijing 2005 experiment were commensurate with the compensation in Zurich and amounted to 4 to 55 Chinese Yuan. Probabilities $p$ of the lotteries' higher gain or loss, $x_{1}$, varied from $5 \%$ to $95 \%$. The gain lotteries for Zurich 2003 are presented in Table 2. The other two experiments essentially included a subset of the Zurich 2003 lotteries. The expected payoff per subject amounted to approximately 31 Swiss Francs and 20 Chinese Yuan, respectively, which was considerably more than a local student assistant's hourly compensation, plus a show up fee of 10 Swiss Francs and 20 Chinese Yuan, thus generating salient incentives. The lotteries appeared in random order on a computer screen ${ }^{3}$, in Beijing on paper.

For each lottery, the screen displayed a decision sheet containing the specifics of the lottery

\footnotetext{
${ }^{1}$ Pooling the data of both treatments does not change the results of our analysis.

${ }^{2}$ At the time of the Zurich 2003 experiment one Swiss Franc equaled about 0.80 U.S. Dollars.

${ }^{3}$ The experiment was programmed and conducted with the software z-Tree (Fischbacher, forthcoming).
} 
Table 2: Gain Lotteries $\left(x_{1}, p ; x_{2}\right)$

\begin{tabular}{ccccccccc}
\hline \hline$p$ & $x_{1}$ & $x_{2}$ & $p$ & $x_{1}$ & $x_{2}$ & $p$ & $x_{1}$ & $x_{2}$ \\
\hline 0.05 & 20 & 0 & 0.25 & 50 & 20 & 0.75 & 50 & 20 \\
0.05 & 40 & 10 & 0.50 & 10 & 0 & 0.90 & 10 & 0 \\
0.05 & 50 & 20 & 0.50 & 20 & 10 & 0.90 & 20 & 10 \\
0.05 & 150 & 50 & 0.50 & 40 & 10 & 0.90 & 50 & 0 \\
0.10 & 10 & 0 & 0.50 & 50 & 0 & 0.95 & 20 & 0 \\
0.10 & 20 & 10 & 0.50 & 50 & 20 & 0.95 & 40 & 10 \\
0.10 & 50 & 0 & 0.50 & 150 & 0 & 0.95 & 50 & 20 \\
0.25 & 20 & 0 & 0.75 & 20 & 0 & & & \\
0.25 & 40 & 10 & 0.75 & 40 & 10 & & & \\
\hline \hline
\end{tabular}

Outcomes $x_{1}$ and $x_{2}$ are denominated in Swiss Francs.

Figure 1: Design of the Decision Sheet

\begin{tabular}{|c|c|c|c|c|c|c|}
\hline \multicolumn{7}{|c|}{$\begin{array}{r}\text { Decision situation: } \\
22 \\
\end{array}$} \\
\hline & Option A & \multicolumn{4}{|c|}{ Your Choice: } & $\begin{array}{c}\text { Option B } \\
\text { Guaranteed payoff amounting to: }\end{array}$ \\
\hline \begin{tabular}{r|}
1 \\
2 \\
3 \\
4 \\
5 \\
6 \\
7 \\
8 \\
9 \\
10 \\
11 \\
12 \\
13 \\
14 \\
15 \\
16 \\
17 \\
18 \\
19 \\
20
\end{tabular} & $\begin{array}{c}\text { A profit of CHF } 20 \text { with } \\
\text { probability } 75 \% \\
\text { and a profit of } \mathrm{CHF} 0 \text { with } \\
\text { probability } 25 \%\end{array}$ & $\begin{array}{l}\mathbf{A} \\
\mathbf{A} \\
\mathbf{A} \\
\mathbf{A} \\
\mathbf{A} \\
\mathbf{A} \\
\mathbf{A} \\
\mathbf{A} \\
\mathbf{A} \\
\mathbf{A} \\
\mathbf{A} \\
\mathbf{A} \\
\mathbf{A} \\
\mathbf{A} \\
\mathbf{A} \\
\mathbf{A} \\
\mathbf{A} \\
\mathbf{A} \\
\mathbf{A} \\
\mathbf{A}\end{array}$ & \begin{tabular}{|l|} 
\\
\\
\\
\\
0 \\
0 \\
0 \\
0 \\
0 \\
0 \\
0 \\
$\circ$ \\
0 \\
0 \\
0 \\
0 \\
0 \\
\end{tabular} & \begin{tabular}{|l|}
0 \\
$\circ$ \\
0 \\
0 \\
0 \\
$\circ$ \\
0 \\
\\
\\
\\
\\
\\
\\
\\
\end{tabular} & $\begin{array}{l}\text { B } \\
\text { B } \\
\text { B } \\
\text { B } \\
\text { B } \\
\text { B } \\
\text { B } \\
\text { B } \\
\text { B } \\
\text { B } \\
\text { B } \\
\text { B } \\
\text { B } \\
\text { B } \\
\text { B } \\
\text { B } \\
\text { B } \\
\text { B } \\
\text { B } \\
\text { B }\end{array}$ & $\begin{array}{l}20 \\
19 \\
18 \\
17 \\
16 \\
15 \\
14 \\
13 \\
12 \\
11 \\
10 \\
9 \\
8 \\
7 \\
6 \\
5 \\
4 \\
3 \\
2 \\
1\end{array}$ \\
\hline \multicolumn{7}{|c|}{ OK } \\
\hline
\end{tabular}


and a list of 20 equally spaced certain outcomes ranging from the lottery's maximum payoff to the lottery's minimum payoff as shown in Figure 1. The subjects had to indicate whether they preferred the lottery or the certain payoff for each line of the decision sheet. The lottery's certainty equivalent was calculated as the arithmetic mean of the smallest certain amount preferred to the lottery and the following certain amount on the list when the subject had for the first time reported preference for the lottery. For example, if the subject had decided as indicated by the small circles in Figure 1 her certainty equivalent would amount to 13.5 Swiss Francs.

Before the subjects were permitted to start working on the experimental decisions they had to correctly calculate the payoffs for two hypothetical choices. In the computerized experiments, there were two trial rounds to familiarize the subjects with the procedure. At the end of the experiment, one of their choices was randomly selected for payment. Subjects were paid in private afterward. The subjects could work at their own speed, the vast majority of them needed less than an hour to complete the experiment.

\section{Econometric Model}

This section discusses the specification of the finite mixture regression model which allows controlling for latent heterogeneity in risk taking behavior in a parsimonious way. Estimating the finite mixture model yields the relative sizes of a pre-specified number of groups and the group-specific parameters of the underlying behavioral model. Moreover, as we use the Expectation Maximization algorithm (Dempster, Laird, and Rubin, 1977) to compute the maximum likelihood estimates of the model parameters we obtain Bayesian updates for the probabilities of individual group membership. This procedure allows us to assign each individual to a specific group.

For our purposes of classifying subjects according to risk taking type we need to specify three ingredients of the mixture model: the basic theory of decision under risk, the functional form of the decision model, and the specification of the error term.

The underlying theory of decision under risk should be able to accommodate a wide range 
of different behaviors. Sign- and rank-dependent models, such as cumulative prospect theory (CPT), capture two robust empirical phenomena: nonlinear probability weighting and loss aversion (Starmer, 2000). Therefore, a flexible approach, such as proposed by CPT, lends itself to describing risk taking behavior ${ }^{4}$. Moreover, CPT nests EUT as special case. If there is a group of people whose behavior can be described by EUT rather than by a non-degenerate version of CPT, these individuals should be identified by the finite mixture regression.

Suppose that there are $C$ different types of individuals in the population. According to CPT, an individual belonging to a certain group $c \in\{1, \ldots, C\}$ values any binary gamble $\mathcal{G}_{g}=\left(x_{1 g}, p_{g} ; x_{2 g}\right), g \in\{1, \ldots, G\}$, where $\left|x_{1 g}\right|>\left|x_{2 g}\right|$, by

$$
v\left(\mathcal{G}_{g}\right)=v\left(x_{1 g}\right) w\left(p_{g}\right)+v\left(x_{2 g}\right)\left(1-w\left(p_{g}\right)\right) .
$$

The function $v(x)$ describes how monetary outcomes, $x$, are valued, whereas the function $w(p)$ assigns a subjective weight to every outcome probability, $p$. The gamble's certainty equivalent $\hat{c e_{g}}$ can then be written as

$$
\hat{c e} e_{g}=v^{-1}\left[v\left(x_{1 g}\right) w\left(p_{g}\right)+v\left(x_{2 g}\right)\left(1-w\left(p_{g}\right)\right)\right] .
$$

In order to make CPT operational, we have to assume specific functional forms for the value function $v(x)$ and the probability weighting function $w(p)$. A natural candidate for $v(x)$ is a sign-dependent power functional

$$
v(x)= \begin{cases}x^{\alpha} & \text { if } x \geq 0 \\ -(-x)^{\beta} & \text { otherwise }\end{cases}
$$

which can be conveniently interpreted and has turned out to be the best compromise between parsimony and goodness of fit in the context of prospect theory (Stott, 2006). The curvature parameters are identifiable since our experimental design contains lotteries with both outcomes being nonzero.

A variety of functionals for modeling probability weights $w(p)$ have been proposed in the literature (Quiggin, 1982; Tversky and Kahneman, 1992; Prelec, 1998). We use the twoparameter specification suggested by Goldstein and Einhorn (1987) and Lattimore, Baker,

\footnotetext{
${ }^{4}$ In the case of binary lotteries, CPT reduces to the original version of prospect theory (Kahneman and Tversky, 1979).
} 
and Witte (1992):

$$
w(p)=\frac{\delta p^{\gamma}}{\delta p^{\gamma}+(1-p)^{\gamma}}, \delta \geq 0, \gamma \geq 0
$$

We favor this specification because it has proven to account well for individual heterogeneity (Wu, Zhang, and Gonzalez, 2004). The parameter $\gamma$ largely governs the slope of the curve, whereas the parameter $\delta$ largely governs its elevation. The smaller the value of $\gamma$, the more strongly the probability weighting function deviates from linear weighting. The larger the value of $\delta$, the more elevated the curve, ceteris paribus. Linear weighting is characterized by $\gamma=\delta=1$. In a sign-dependent model, the parameters may take on different values for gains and for losses.

We now turn to the third step of model specification. In the course of the experiments, we measured risk taking behavior of individual $i \in\{1, \ldots, N\}$ by her certainty equivalents $c e_{i g}$ for a set of different lotteries. Since CPT explains deterministic choice we have to add an error term, $\epsilon_{i g}$, in order to estimate the parameters of the model based on the elicited certainty equivalents. The observed certainty equivalent $c e_{i g}$ can then be written as $c e_{i g}=\hat{c} e_{g}+\epsilon_{i g}$. There may be different sources of error, such as carelessness, hurry or inattentiveness, resulting in accidentally wrong answers (Hey and Orme, 1994). The Central Limit Theorem supports the assumption that the errors are normally distributed and simply add white noise.

Furthermore, we allow for three different sources of heteroscedasticity in the error variance. First, for each lottery the subjects have to consider 20 certain outcomes which are equally spaced throughout the lottery's range, $\left|x_{1 g}-x_{2 g}\right|$. Since the observed certainty equivalent, $c e_{i g}$, is calculated as the arithmetic mean of the smallest certain amount preferred to the lottery and the following certain amount where the lottery is preferred, the error is proportional to the range of the considered lottery. Second, as the subjects are heterogeneous with respect to their previous knowledge, their ability of finding the correct certainty equivalent as well as their attention span we expect the error variance to differ by individual. Third, lotteries in the gain domain may be judged differently from the ones in the loss domain. Therefore we allow for domain-specific variance in the error term. This yields the form $\sigma_{i g}=\xi_{i}\left|x_{1 g}-x_{2 g}\right|$ for the standard deviation of the error term distribution, where $\xi_{i}$ denotes an individual domain- 
specific parameter. Note that the model allows to test for both individual-specific and domainspecific heteroscedasticity by either imposing the restriction $\xi_{i}=\xi$, or by forcing all the $\xi_{i}$ to be equal in both decision domains. Both restrictions are rejected by their corresponding likelihood ratio tests in all three samples with p-values close to zero. Therefore we control for all three types of heteroscedasticity in the estimation procedure.

Having discussed all the necessary ingredients we now turn to the specification of the finite mixture regression model. The basic idea of the mixture model is assigning an individual's risk-taking choices to one of $C$ different types of behavior each characterized by a distinct vector of parameters $\theta_{c}=\left(\alpha_{c}, \beta_{c}, \gamma_{c}^{\prime}, \delta_{c}^{\prime}\right)^{\prime 5}$. We denote the proportions of these different types in the population by $\pi_{c}$. Given our assumptions on the distribution of the error term, the density of type $c$ can be expressed as

$$
f\left(c e_{i}, \mathcal{G} ; \theta_{c}, \xi_{i}\right)=\prod_{g=1}^{G} \frac{1}{\sigma_{i g}} \phi\left(\frac{c e_{i g}-\hat{c e} e_{g}}{\sigma_{i g}}\right)
$$

for the $i$-th individual, where $\phi(\cdot)$ denotes the density of the standard normal distribution. Since we do not know a priori to which group a certain individual belongs to, the proportions $\pi_{c}$ are interpreted as probabilities of group membership. Therefore, each individual density of type $c$ has to be weighted by its respective mixing proportion $\pi_{c}$ which, of course, is unknown and has to be estimated as well. Summing over all $C$ components yields the individual's contribution to the model's likelihood function $L$. The log likelihood of the finite mixture regression model is then given by

$$
\ln L(\Psi ; c e, \mathcal{G})=\sum_{i=1}^{N} \ln \sum_{c=1}^{C} \pi_{c} f\left(c e_{i}, \mathcal{G} ; \theta_{c}, \xi_{i}\right),
$$

where the vector $\Psi=\left(\theta_{1}^{\prime}, \ldots, \theta_{C}^{\prime}, \pi_{1}, \ldots, \pi_{C-1}, \xi_{1}, \ldots, \xi_{N}\right)^{\prime}$ summarizes all the parameters of the model which need to be estimated.

For estimating the model we use the iterative Expectation Maximization (EM) algorithm which provides an additional feature: By Bayesian updating, the algorithm calculates in each iteration an individual's posterior probability $\tau_{i c}$ of belonging to group $c$. The posterior

\footnotetext{
${ }^{5}$ The vectors $\gamma_{c}$ and $\delta_{c}$ contain the domain-specific parameters for the slope and the elevation of the probability weighting functions.
} 
probabilities $\tau_{i c}$ represent a particularly valuable result of the estimation procedure. Not only do we obtain the probabilities of individual group membership but we also have a method of judging the quality of the classification at our disposal. If all the $\tau_{i c}$ are either close to zero or one all the individuals are unambiguously assigned to one specific group. The $\tau_{i c}$ can be used to calculate a summary measure of ambiguity, such as the average normalized entropy (El-Gamal and Grether, 1995), in order to gage the extent of dubious assignments and to discriminate between models with differing numbers of types. If the classification has been successful we should observe a low measure of entropy. For example, if entropy increases when the number of different types is increased from two to three, the group assignment of the individuals is less reliable and the model tends to overfit the data. Therefore, the model with two types is to be preferred. Thus, the entropy measure enables us to determine the optimal number of distinct types.

We will briefly illustrate the intuition of the iterative estimation routine: Suppose that there are several different types of individuals in the population, each characterized by a distinct set of parameter values. If individual group membership were known, the estimates for the relative group sizes $\pi_{c}$ would be the relative number of individuals in the respective group and the group-specific parameter values $\theta_{c}$ could be obtained by separately maximizing the joint density function of the respective group. However, as we cannot observe individual group membership directly we face an incomplete data problem and the direct maximization of the model's likelihood function would be difficult (for details see the Appendix). The EM algorithm now proceeds iteratively in two steps: First, by Bayesian updating an individual's posterior probability $\tau_{i c}$ of belonging to a specific group $c$ is obtained based on the actual fit of the data. As these $\tau_{i c}$ provide an estimate for unobserved individual group membership, they can be used in the second step of the iteration to estimate a new set of model parameters. As Dempster, Laird, and Rubin (1977) have shown, this procedure results in an increase of the likelihood value in each iteration.

Various problems may be encountered when maximizing the likelihood function of a finite mixture regression model and, therefore, a customized estimation procedure has to be used which can adequately deal with these problems. Details of the estimation procedure, written 
in the $R$ environment ( $\mathrm{R}$ Development Core Team, 2006), are discussed in the Appendix.

\section{Results}

In the following section we describe observed risk taking behavior and present the results of the finite mixture regression model. We discuss the quality of the classification procedure and the number of heterogeneous groups identified in the data. Finally, we characterize the representative types found in each data set by their behavioral parameters and discuss crosscultural differences.

RESULT 1: At the aggregate level, the data exhibit the fourfold pattern of risk attitudes predicted by CPT, i.e. subjects exhibit risk aversion for high-probability gains and low-probability losses, and risk seeking for low-probability gains and high-probability losses.

Support. Observed risk taking behavior can be conveniently summarized by relative risk premia $R R P=(e v-c e) /|e v|$, where $e v$ denotes the expected value of a lottery's payoff and ce stands for its certainty equivalent. $R R P>0$ indicates risk aversion, $R R P<0$ risk seeking, and $R R P=0$ risk neutrality. In the context of EUT, risk preferences are captured solely by the curvature of the utility function which in turn determines the sign of the relative risk premium. Therefore, the sign of $R R P$ should be independent of $p$, the probability of the more extreme lottery outcome. In Figures 2 through 4 , median risk premia sorted by $p$ show a systematic relationship between $R R P$ and $p$, however: In all three data sets subjects' choices display a fourfold pattern, i.e. they are risk averse for low-probability losses and highprobability gains, and they are risk seeking for low-probability gains and high-probability losses. Therefore, at a first glance, average behavior is adequately described by a model such as CPT rather than EUT.

The median RRPs gloss over an important feature of the data, however: There is substantial latent heterogeneity in risk taking behavior which is uncovered by the finite mixture regression.

RESULT 2: The heterogeneity of individuals' risk preferences can be captured by two distinct types of behavior. Assuming three distinct types yields an inferior characterization of the 
Figure 2: Median Relative Risk Premia Zurich 2003
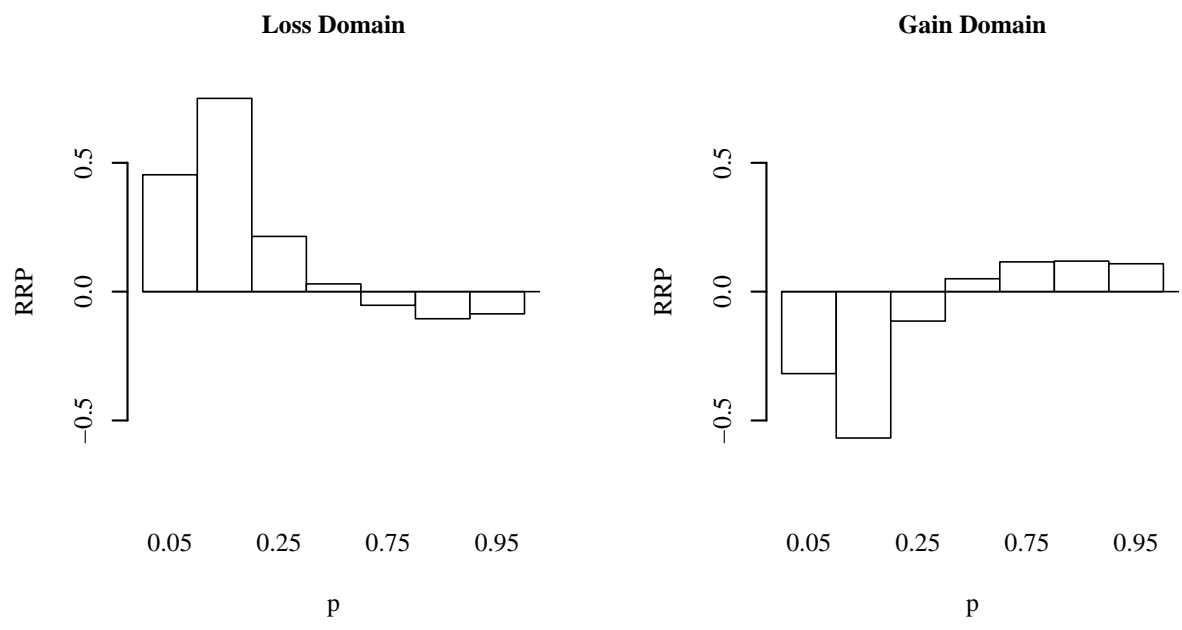

Figure 3: Median Relative Risk Premia Zurich 2006
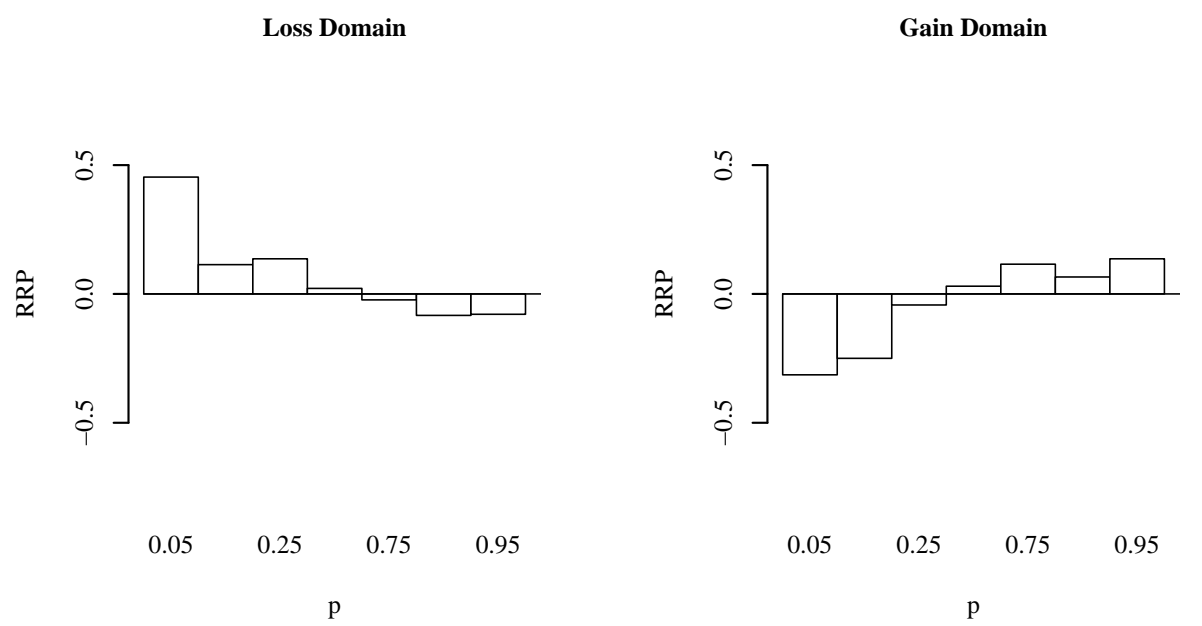
Figure 4: Median Relative Risk Premia Beijing 2005

Loss Domain

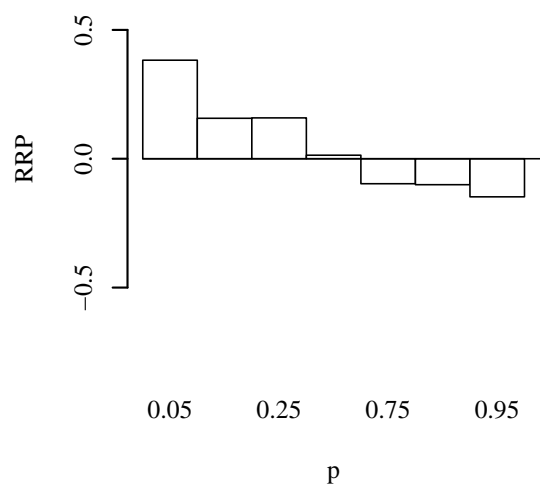

Gain Domain

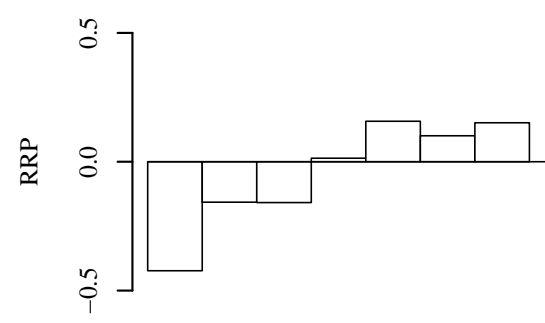

underlying heterogeneity.

Support. The finite mixture regression model classifies individuals according to a given number of types. In order to evaluate the quality of classification, we calculated the average normalized entropy ANE (El-Gamal and Grether, 1995) defined as

$$
A N E=-\frac{1}{N} \sum_{i=1}^{N} \sum_{c=1}^{C} \tau_{i c} \log _{C}\left(\tau_{i c}\right),
$$

for $C$ groups and $N$ individuals. Taking $\log _{C}$ normalizes the entropy measure to lie within $[0,1]$. If all the $\tau_{i c}$ are equal to zero or one, $A N E=0$. In this case, all the individuals can be perfectly assigned to one group. $A N E=1$ reflects maximum entropy, i.e. all the $\tau_{i c}$ are equal to $1 / C$. Such a result indicates that group membership is totally ambiguous and that categorization has failed. If this were the case, the model's assumption that there is a specific number of distinct types in the population could be refuted and, thus, using a finite mixture regression model would be inappropriate. The first line in Table 3 displays the average normalized entropy for two groups. All three data sets exhibit an average entropy of less than $5 \%$ of the maximal entropy of one which is an extremely low degree of ambiguity by any standard. In their experiment on Bayesian learning, for instance, El-Gamal and Grether 
(1995) find the average entropy to lie between 0.11 and 0.38 which they interpret to be "quite small".

These low values of $A N E$ in our analysis indicate that nearly all the individuals can be unambiguously assigned to one of the two groups. This result can also be inferred from the distributions of the posterior probabilities of group assignment in Figure 5 . In Figure $5 \tau_{E U T}$ denotes the posterior probability of belonging to the first group which can be characterized, as we will demonstrate below, as expected utility maximizers. In all three data sets, the individuals' posterior probability of being an expected utility maximizer is either close to one or close to zero for practically all the individuals. Our result is quite remarkable as it substantiates that there are two distinct types in the population and not a continuity of heterogeneous individuals. And it also shows that the underlying behavioral model provides a sound basis of discriminating between types.

Given the extremely low degree of ambiguity in our two-group classification, an improvement in entropy when three groups are assumed seems hardly possible. If the classification procedure worked better for three groups than for two groups, the average normalized entropy should be smaller for $C=3$ than for $C=2$. Table 3 shows that this is not the case in any of the three data sets. So we can safely conclude that two groups are sufficient to capture the essential characteristics of individual heterogeneity in risk taking behavior.

Table 3: Average Normalized Entropy

\begin{tabular}{lccc}
\hline \hline Groups & Zurich 03 & Zurich 06 & Beijing 05 \\
\hline$C=2$ & 0.049 & 0.033 & 0.031 \\
$C=3$ & 0.052 & 0.034 & 0.049 \\
\hline \hline
\end{tabular}

Aside from the high reliability of assignment, we also find stable mixing proportions across all three data sets as the next result shows.

RESULT 3: The proportions of the two distinct types are essentially equal in all three data sets and amount to a ratio of approximately 20:80. 
Figure 5: Distribution of Posterior Probability of Assignment to EUT

Zurich 2003

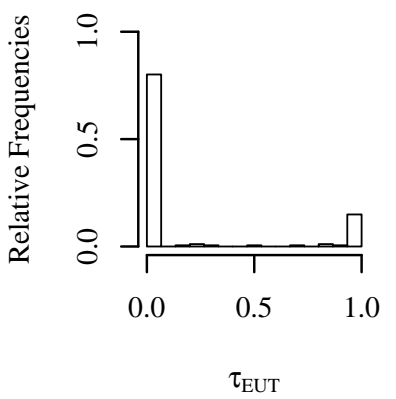

Zurich 2006

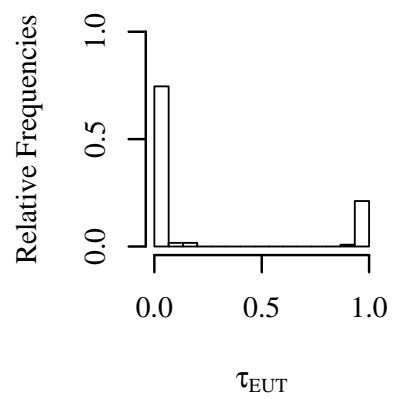

Beijing 2005

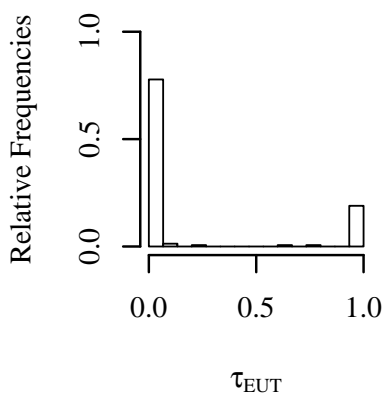

Support. In all three graphs of Figure 5, there are about four times as many individuals with $\tau_{E U T}$ close to zero as individuals with $\tau_{E U T}$ close to one. This finding is mirrored by the estimates of the mixing proportions $\pi_{c}$. Table 4 displays, for each data set, the group-specific parameter estimates of the finite mixture regression model and their standard errors obtained by the bootstrap method with 4,000 replications (Efron and Tibshirani, 1993). Estimates of the mixing proportion of the first group amount to about $20 \%$ and, consequently, to about $80 \%$ for the second group. Moreover, the $95 \%$-confidence intervals for the estimates of $\pi_{c}$ for all three data sets overlap. Therefore, the classification is not only unambiguous but also results in roughly equal proportions of both types across our data sets.

This finding leads us to the next question. Can each of the two types found be characterized by essentially the same patterns of behavior across all three data sets?

RESULT 4: One type of individual behavior, applying to approximately 20\% of the subjects, is characterized by near linear probability weighting and linear valuation of monetary outcomes. Thus, these individuals behave in accordance with expected value maximization.

Support. As far as the first type is concerned, which comprises about $20 \%$ of the subjects, Table 4 displays almost identical parameter estimates across all three data sets. Without having imposed any restrictions on the parameters, we find that the first groups' probability weighting functions are roughly linear as the parameter estimates for both $\gamma$ and $\delta$ are close to 
Table 4: Classification of Behavior

\begin{tabular}{|c|c|c|c|c|c|c|}
\hline \multirow[b]{2}{*}{ Parameters } & \multicolumn{3}{|c|}{ EUT-Types } & \multicolumn{3}{|c|}{ СРТ-Тypes } \\
\hline & Zurich 03 & Zurich 06 & Beijing 05 & Zurich 03 & Zurich 06 & Beijing 05 \\
\hline \multirow[t]{2}{*}{ Proportion $\pi$} & 0.176 & 0.224 & 0.201 & 0.824 & 0.776 & 0.799 \\
\hline & $(0.022)$ & $(0.026)$ & $(0.020)$ & $(0.022)$ & $(0.026)$ & $(0.020)$ \\
\hline \multicolumn{7}{|l|}{ Gains } \\
\hline \multirow[t]{2}{*}{$\alpha$} & 0.983 & 0.989 & 1.083 & 1.056 & 0.901 & 0.379 \\
\hline & $(0.012)$ & $(0.018)$ & $(0.103)$ & $(0.021)$ & $(0.026)$ & $(0.105)$ \\
\hline \multirow[t]{2}{*}{$\gamma$} & 0.952 & 0.945 & 0.911 & 0.414 & 0.425 & 0.242 \\
\hline & $(0.014)$ & $(0.020)$ & $(0.034)$ & $(0.015)$ & $(0.015)$ & $(0.014)$ \\
\hline \multirow[t]{2}{*}{$\delta$} & 0.907 & 0.909 & 0.889 & 0.846 & 0.862 & 1.335 \\
\hline & $(0.012)$ & $(0.019)$ & $(0.054)$ & $(0.021)$ & $(0.028)$ & $(0.074)$ \\
\hline \multicolumn{7}{|l|}{ Losses } \\
\hline \multirow[t]{2}{*}{$\beta$} & 1.009 & 1.014 & 1.020 & 1.108 & 1.121 & 1.156 \\
\hline & $(0.017)$ & $(0.024)$ & $(0.087)$ & $(0.027)$ & $(0.047)$ & $(0.108)$ \\
\hline \multirow[t]{2}{*}{$\gamma$} & 0.871 & 0.953 & 0.948 & 0.417 & 0.452 & 0.306 \\
\hline & $(0.042)$ & $(0.020)$ & $(0.040)$ & $(0.016)$ & $(0.014)$ & $(0.013)$ \\
\hline \multirow[t]{2}{*}{$\delta$} & 0.966 & 1.049 & 1.066 & 1.021 & 1.060 & 0.925 \\
\hline & $(0.059)$ & $(0.033)$ & $(0.066)$ & $(0.027)$ & $(0.044)$ & $(0.054)$ \\
\hline $\ln L$ & 20,493 & 11,336 & 10,244 & & & \\
\hline Parameters & 375 & 249 & 319 & & & \\
\hline Observations & 9,005 & 4,669 & 4,281 & & & \\
\hline
\end{tabular}

Standard errors (in parentheses) are based on the bootstrap method with 4,000 replications.

Parameters include additional estimates for $\hat{\xi}_{i}$ for domain- and individual-specific error variances. 
Figure 6: Probability Weighting Functions Zurich 2003
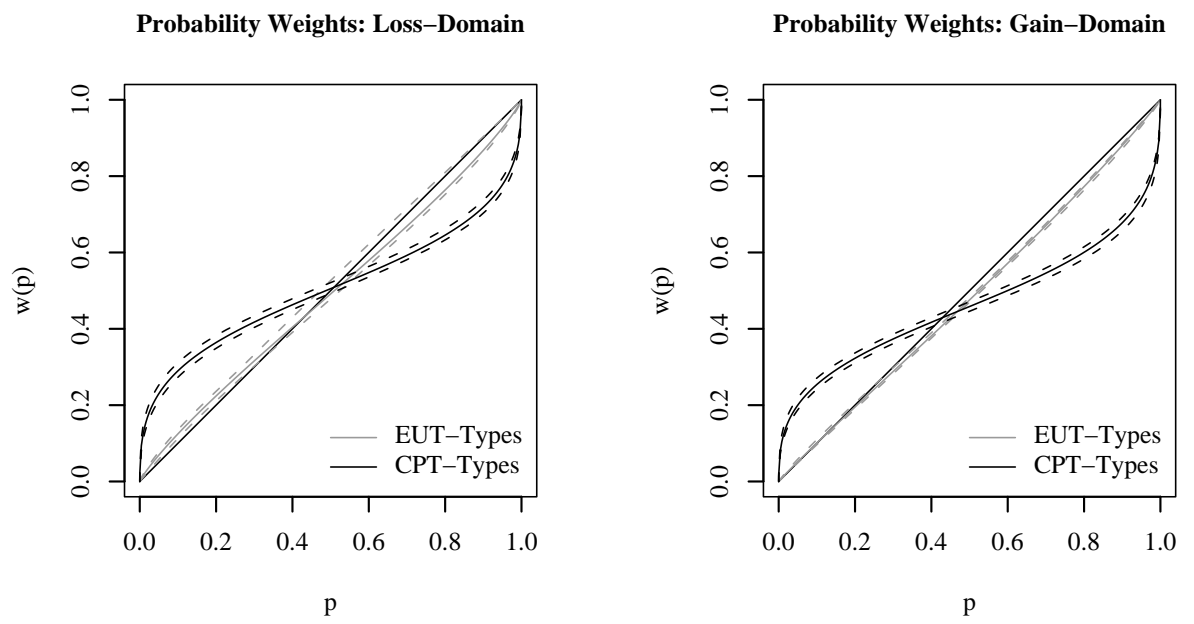

Figure 7: Probability Weighting Functions Zurich 2006

Probability Weights: Loss-Domain

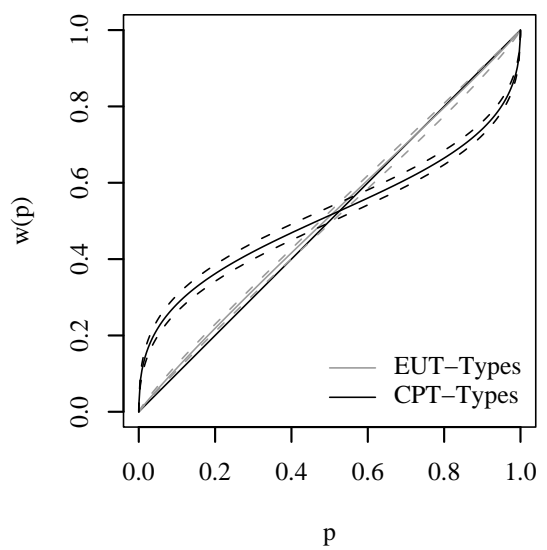

Probability Weights: Gain-Domain

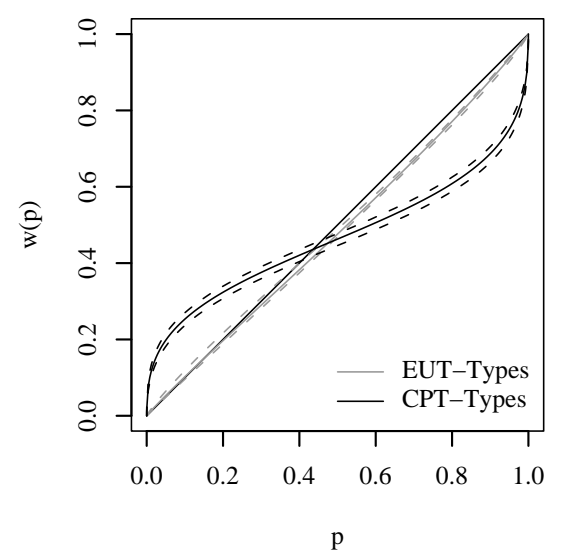


Figure 8: Probability Weighting Functions Beijing 2005
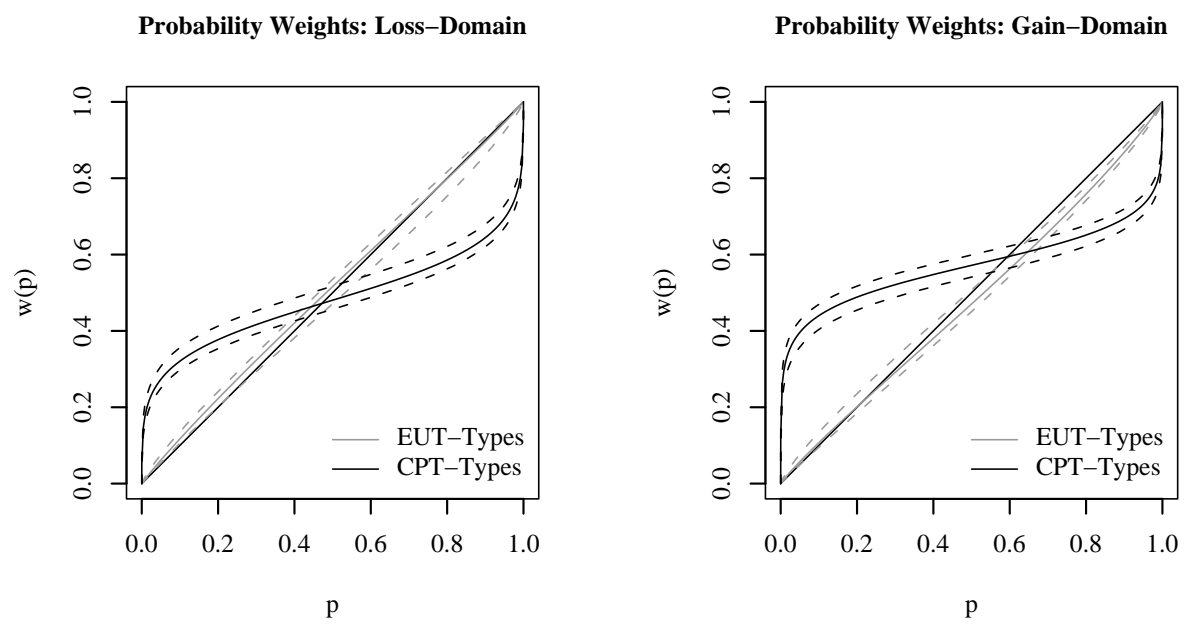

one and, in many of the cases, cannot be statistically distinguished from one. The confidence bands for the probability weighting functions reveal the same picture: Figures 6, 7, and 8 contain the graphs of the domain-specific probability weighting functions by type with their confidence bands based on the percentile bootstrap method. The gray dotted lines correspond to the estimated curves for the first type, referred to as "EUT-type", the gray dashed lines delimit the respective confidence bands. For both gains and losses, the confidence bands for the first type include linear weighting over a wide range of probabilities. Moreover, as Table 4 reveals, the estimates for the power functional parameters $\alpha$ and $\beta$ are also practically equal to one, so these groups can be essentially characterized as expected value maximizers. In the light of Rabin's calibration theorem, we label the individuals belonging to these groups as "EUT-types".

The discriminatory power of our classification can also be traced at the behavioral level. After assigning the subjects to one of the two groups based on their $\tau_{i c}$, the observed relative risk premia can be broken down by type as depicted in Figure 9 for the Chinese data set. As can be seen, the RRPs of the Chinese EUT-types are close to zero, reflecting near risk neutral behavior in accordance with expected value maximization. A similar picture can be shown to 
emerge for the Zurich 2003 and Zurich 2006 experiments.

The next result characterizes the second group of individuals. The observed fourfold pattern of risk attitudes, depicted in Figures 2 through 4, already suggests that nonlinear probability weighting is a dominant feature of aggregate behavior.

RESULT 5: The second type of individuals, comprising about $80 \%$ of the subjects, exhibit an inverted $S$-shaped probability weighting curve consistent with CPT.

Support. The second, much larger, class of individuals in each data set is characterized by a typical inverted S-shaped probability weighting function. Consequently, we label these individuals as "CPT-types". The CPT-types' probability weighting curves are pictured as black lines in Figures 6, 7, and 8. The solid lines correspond to the estimated curves and the dashed lines mark the confidence bands. For both gains and losses, all three figures show nonlinear probability weighting curves. Examining the behavior of the Chinese CPT-types at the level of observed relative risk premia in Figure 9, we find a pronounced fourfold pattern of risk attitudes with more extreme deviations from risk neutrality than the aggregate risk premia in Figure 4. As before, a similar picture can be shown to emerge for the Zurich 2003 and Zurich 2006 data. This finding demonstrates that aggregate data underestimate the true extent of the CPT-types' probability distortions.

Across all three data sets we found a surprisingly similarity of behavior of the first group, the EUT-types. Is the second groups' behavior also devoid of cultural specificities?

RESULT 6: A cross-cultural difference in the CPT-types' behavior is manifest in the gaindomain whereas in the loss domain parameter estimates exhibit consistent magnitudes across all three data sets. For gains, the Chinese subjects weight probabilities more optimistically than the Swiss subjects. Moreover, they exhibit a clearly concave value function whereas the Swiss value functions are near linear.

Support. Inspection of the parameter estimates in Table 4 reveals the values for the loss domain to be essentially of the same order of magnitude across all three data sets: All the estimates for the curvature parameter $\beta$ are close to 1.1, the estimated slope parameter $\gamma$ of the probability weighting function lies between 0.31 and 0.45 , and the elevation parameter estimates are in the vicinity of 1 . The graphs of the CPT-types' probability weighting functions 
Figure 9: Median Relative Risk Premia by Type Beijing 2005

Loss Domain: Prospect Theory Types

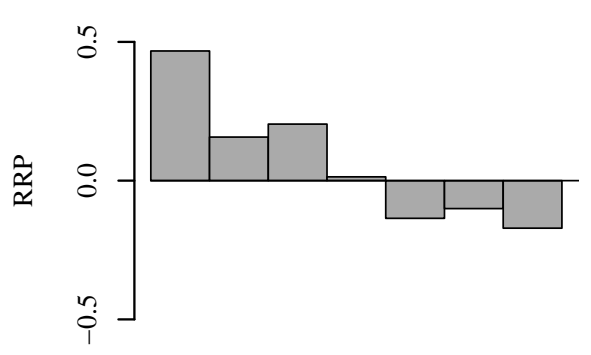

0.05

0.25

0.75

$\mathrm{p}$

Loss Domain: Expected Utility Maximizers

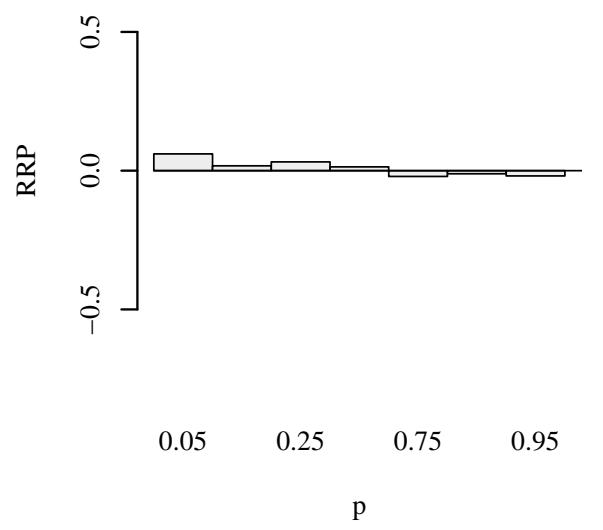

Gain Domain: Prospect Theory Types

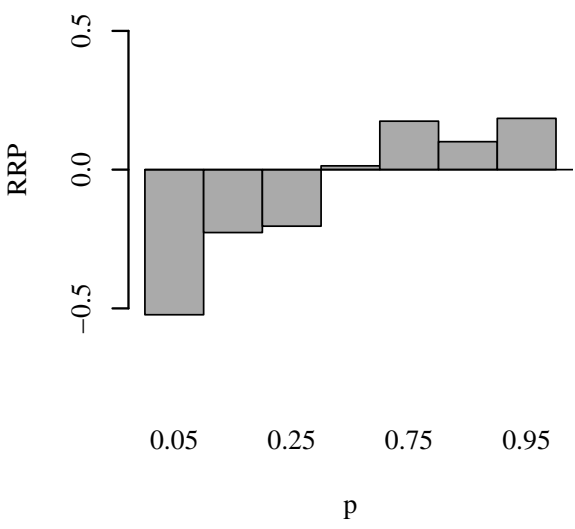

Gain Domain: Expected Utility Maximizers

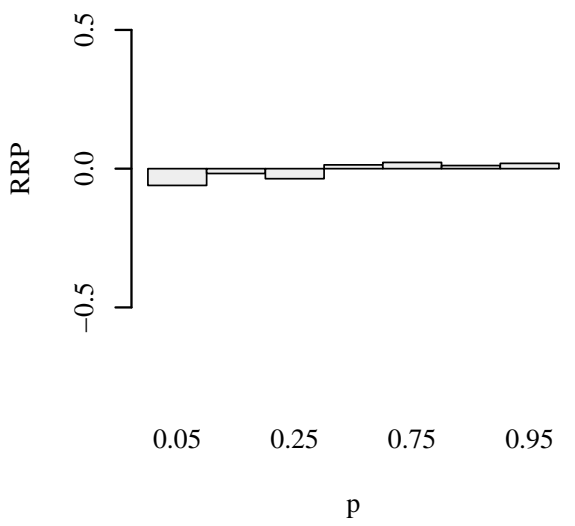


over losses all look similar when comparing Figures 6, 7, and 8. We find a substantial cultural difference between Swiss and Chinese subjects in the gain domain, however. As the graphs show, the Swiss probability weighting functions in Figures 6 and 7 exhibit the familiar shape, i.e. intersection with the diagonal at probabilities of about 0.4 , whereas the Chinese curve in Figure 8 is much more elevated. When judging gains, the Chinese subjects seem to be much more optimistic than the Swiss, i.e. they put a much higher weight on small and medium probabilities. The Chinese probability weighting function is also considerably flatter in the middle part than the Swiss curves which indicates a lower sensitivity towards changes in probabilities by the Chinese. On the other hand, the Chinese value function is clearly concave (estimated $\alpha$ equals 0.38), contrasting with the almost linear Swiss value functions (estimated $\alpha$ equals 1.06 and 0.90 , respectively).

\section{Concluding Remarks}

We conducted three experiments based on the same design principles and applied a finite mixture regression model to the resulting data. For all three data sets a coherent picture emerges. Irrespective of cultural background, we find an equal mix of two distinct groups. The classification procedure performs extremely well resulting in less than $5 \%$ of the maximal average normalized entropy which means that almost all the individuals are reliably assigned to either one of the two distinct groups. The first group comprises about $20 \%$ of the subjects, be they Swiss or Chinese, whose behavior can be characterized by expected utility theory. Moreover, parameter estimates are almost identical for all three EUT-groups and correspond to near risk neutral behavior in line with the prediction of Rabin's calibration theorem.

The second group, encompassing $80 \%$ of the subjects, can be classified as prospect theory types exhibiting an inverted S-shaped probability weighting function. When potential losses are at stake, the behavior of CPT-types can be described by remarkably similar parameter values. In the domain of gains, however, we find significant cross-cultural differences. Chinese risk taking behavior can be explained by two countervailing forces: While probabilities are weighted highly optimistically, the marginal value of monetary outcomes declines. The risk 
attitude of the Swiss subjects, however, manifests itself mostly in the shape of the probability weighting function alone. When we estimate risk premia over a comparable range of outcomes, we predict the Chinese to be more risk seeking than the Swiss for gains of low and medium probability. Previous studies show that Chinese respondents are relatively more risk seeking than American respondents (Hsee and Weber, 1999; Wang and Fischbeck, 2004). These results are consistent with our estimates and can be explained predominantly by the specific shape of the Chinese probability weighting function.

When we started this project we were quite confident that we would find a considerable fraction of rational expected utility maximizers. What really surprised us is the robust share of EUT-types, even across two so different cultures as the Swiss and Chinese. This consistent magnitude of the EUT-groups lends support to prior evidence by Hey and Orme (1994) and Lattimore, Baker, and Witte (1992). These rational actors constitute a non-negligible proportion of the population whose behavior, depending on the nature of the strategic environment, may be decisive for aggregate outcomes. The existence of a robust share of rational actors suggests to use a mix of preference theories for modeling behavior rather than a single theory which would yield systematically biased results. Moreover, for the majority of subjects, prospect theory adequately describes behavior, but the parameter estimates exhibit culturespecific values. Researchers should take this evidence into account when constructing and estimating models of choice under risk.

ACKNOWLEDGMENTS: We would like to thank Ernst Fehr, Rainer Winkelmann, and Michael Wolf for their valuable comments. 


\section{References}

Camerer, C. F., And E. Fehr (2006): "When Does Economic Man Dominate Social Behavior?," Science, 311.

Celeux, G., D. Chauveau, and J. Diebolt (1995): "On Stochastic Version of the EM Algorithm," Penn State University, Working Paper, (RR-2514), 22 p.

Dempster, A., N. Laird, and D. Rubin (1977): "Maximum Likelihood from Incomplete Data via the EM Algorithm," Journal of the Royal Statistical Society, Series B, 39(1), 1-38.

Dohmen, T., A. Falk, D. Huffman, U. Sunde, J. Schupp, and G. G. Wagner (2005): "Individual Risk Attitudes: New Evidence from a Large, Representative, ExperimentallyValidated Survey," Institute for the Study of Labor (IZA), Bonn (Germany), Discussion Paper, 1730.

Efron, B., And R. J. Tibshirani (1993): An Introduction to the Bootstrap. Chapman \& Hall/CRC, ISBN 0-412-04231-2.

El-Gamal, M. A., And D. M. Grether (1995): "Are People Bayesian? Uncovering Behavioral Strategies," Journal of the American Statistical Association, 90(432), 1137-1145.

FeHR, E., And J.-R. TyRAn (2005): "Individual Irrationality and Aggregate Outcomes," Journal of Economic Perspectives, 19(4).

Fehr-Duda, H., M. de Gennaro, and R. Schubert (2006): "Gender, Financial Risk, and Probability Weights," Theory and Decision, 60, 283-313.

Fischbacher, U. (forthcoming): "z-Tree: Zurich Toolbox for Readymade Economic Experiments," Experimental Economics.

Goldstein, W., and H. Einhorn (1987): "Expression Theory and the Preference Reversal Phenomena," Psychological Review, 94, 236-254. 
Haltiwanger, J. C., and M. Waldman (1985): "Rational Expectations and the Limits of Rationality: An Analysis of Heterogeneity," American Economic Review, 75(3), 326-340.

(1989): "Limited Rationality and Strategic Complements: The Implications for Macroeconomics," Quarterly Journal of Economics, 104(3), 463-483.

Harless, D. W., And C. F. Camerer (1994): "The Predictive Utility of Generalized Expected Utility Theories," Econometrica, 62, 1251-1290.

Harrison, G. W., S. J. Humphrey, and A. Verschoor (2005): "Choice Under Uncertainty in Developing Countries," Department of Economics, College of Business Administration, University of Central Florida, Working Paper, 0529.

Harrison, G. W., and E. E. Rutstroem (2006): "Expected Utility Theory and Prospect Theory: One Wedding and A Decent Funeral," Department of Economics, College of Business Administration, University of Central Florida, Working Paper, 0518.

HeY, J. D., And C. Orme (1994): "Investigating Generalizations of Expected Utility Theory Using Experimental Data," Econometrica, 62(6), 1291-1326.

Houser, D., M. Keane, and K. McCabe (2004): "Behavior in a Dynamic Decision Problem: An Analysis of Experimental Evidence using a Bayesian Type Classification Algorithm," Econometrica, 72(3), 781-822.

Hsee, C. K., And E. U. Weber (1999): "Cross-National Differences in Risk Preferences and Lay Predictions," Journal of Behavioral Decision Making, 12, 165-179.

Kahneman, D., and A. Tversky (1979): "Prospect Theory: An Analysis of Decision under Risk," Econometrica, 47(2), 263-292.

Lattimore, P. K., J. R. Baker, and A. D. Witte (1992): "The Influence of Probability on Risky Choice," Journal of Economic Behavior and Organization, 17, 377-400.

McLachlan, G., and D. Peel (2000): Finite Mixture Models. Wiley Series in Probabilities and Statistics. 
Prelec, D. (1998): "The Probability Weighting Function," Econometrica, 3, 497-527.

Quigain, J. (1982): "A Theory of Anticipated Utility," Journal of Economic Behavior and Organization, 3, 323-343.

R Development Core Team (2006): "R: A Language and Environment for Statistical Computing," R Foundation for Statistical Computing (Vienna, Austria), ISBN 3-90005107-0, http://www.R-project.org.

Rabin, M. (2000): "Risk Aversion and Expected-Utility Theory: A Calibration Theorem," Econometrica, 68(5), 1281-1292.

Render, R. A., and X. Walker (1984): "Mixture Densities, Maximum Likelihood and the EM Algorithm," SIAM Review, 26(2), 195-239.

Starmer, C. (2000): "Developments in Non-Expected Utility Theory: The Hunt for a Descriptive Theory of Choice under Risk," Journal of Economic Literature, 38(2), 332-382.

Stотт, H. P. (2006): "Cumulative Prospect Theory's Functional Menagerie," Journal of Risk and Uncertainty, 32, 101-130.

Tversky, A., and D. Kahneman (1992): "Advances in Prospect Theory: Cumulative Representation of Uncertainty," Journal of Risk and Uncertainty, 5, 297-323.

Wang, M., And P. S. Fischbeck (2004): "Incorporating Framing into Prospect Theory Modeling: A Mixture-Model Approach," Journal of Risk and Uncertainty, 29(2), 181-197.

Wu, G., J. Zhang, and R. Gonzalez (2004): Decision under Risk. D. Koehler and N. Harvey (Eds.), The Blackwell Handbook of Judgment and Decision Making, Oxford University Press. 


\section{A Estimation of the Finite Mixture Regression Model}

As it is generally the case in finite mixture models, direct maximization of the log likelihood function

$$
\ln L(\Psi ; c e, \mathcal{G})=\sum_{i=1}^{N} \ln \sum_{c=1}^{C} \pi_{c} f\left(c e_{i}, \mathcal{G} ; \theta_{c}, \xi_{i}\right)
$$

may encounter several problems, even if it is in principle feasible (for a general treatise see for example McLachlan and Peel (2000)). First, the highly non-linear form of the log likelihood causes the optimization algorithm to be rather slow or even incapable of finding the maximum. Second, the likelihood of a finite mixture model is often multimodal and therefore we have no guaranty that a standard optimization routine will converge towards the global maximum rather than to one of the local maxima.

However, if individual group-membership were observable and indicated by $t_{i c} \in\{0,1\}$ the individual contribution to the likelihood function would be given by

$$
\tilde{\ell}\left(\Psi_{i} ; c e_{i}, \mathcal{G}, t_{i}\right)=\prod_{c=1}^{C}\left[\pi_{c} f\left(c e_{i}, \mathcal{G} ; \theta_{c}, \xi_{i}\right)\right]^{t_{i c}}
$$

By using the above formulation and taking logarithms, the complete-data log likelihood function

$$
\ln \tilde{L}(\Psi ; c e, \mathcal{G}, t)=\sum_{i=1}^{N} \sum_{c=1}^{C} t_{i c}\left[\ln \pi_{c}+\ln f\left(c e_{i}, \mathcal{G} ; \theta_{c}, \xi_{i}\right)\right]
$$

would follow directly. As relative group sizes sum up to one, their maximum likelihood estimates, $\hat{\pi}_{c}=1 / N \sum_{i=1}^{N} t_{i c}$, would be given analytically by the relative number of individuals in the respective group. Furthermore, the maximum likelihood estimates of the group-specific parameters could be obtained separately in each group by numerically maximizing the corresponding joint density function which would simplify the optimization problem considerably.

The EM algorithm proceeds iteratively in two steps, E and M, while it treats the unobservable $t_{i c}$ as missing data. In the E-step of the $(k+1)$-th iteration the expectation of the

complete-data $\log$ likelihood $\tilde{L}$, given the actual fit of the data $\Psi^{(k)}$, is computed. This yields, 
according to Bayes' law, the posterior probabilities of individual group-membership

$$
\tau_{i c}\left(c e_{i}, \mathcal{G} ; \Psi_{i}^{(k)}\right)=\frac{\pi_{c}^{(k)} f\left(c e_{i}, \mathcal{G} ; \theta_{c}^{(k)}, \xi_{i}^{(k)}\right)}{\sum_{m=1}^{C} \pi_{m}^{(k)} f\left(c e_{i}, \mathcal{G} ; \theta_{m}^{(k)}, \xi_{i}^{(k)}\right)}
$$

which replace the unknown indicators of individual group-membership, $t_{i c}$. Given $\tau_{i c}\left(c e_{i}, \mathcal{G} ; \Psi_{i}^{(k)}\right)$, the complete-data $\log$ likelihood, $\tilde{L}$, is maximized in the following M-step which yields the updates of the model parameters,

$$
\pi_{c}^{(k+1)}=\frac{1}{N} \sum_{i=1}^{N} \tau_{i c}\left(c e_{i}, \mathcal{G} ; \Psi_{i}^{(k)}\right),
$$

and

$$
\begin{aligned}
& \left(\theta_{1}^{(k+1)}, \ldots, \theta_{C}^{(k+1)}, \xi_{1}^{(k+1)}, \ldots, \xi_{N}^{(k+1)}\right)= \\
& \underset{\theta_{1}, \ldots, \theta_{C}, \xi_{1}, \ldots, \xi_{N}}{\arg \max } \sum_{i=1}^{N} \sum_{m=1}^{C} \tau_{i m}\left(c e_{i}, \mathcal{G} ; \Psi_{i}^{(k)}\right) \ln f\left(c e_{i}, \mathcal{G} ; \theta_{m}^{(k)}, \xi_{i}^{(k)}\right) .
\end{aligned}
$$

As Dempster, Laird, and Rubin (1977) show, the likelihood never decreases from one iteration to the next, i.e. $L\left(\Psi^{(k+1)} ; c e, \mathcal{G}\right) \geq L\left(\Psi^{(k)} ; c e, \mathcal{G}\right)$, which makes the EM algorithm converge monotonically towards the nearest maximum of the likelihood function regardless whether this maximum is global or just local. In the Zurich 2003 data set, we therefore needed to apply a stochastic extension, the Simulated Annealing Expectation Maximization (SAEM) algorithm proposed by Celeux, Chauveau, and Diebolt (1995), in order to overcome the EM algorithm's tendency to converge towards local maxima. In each iteration, there is a non-zero probability that the SAEM algorithm leaves the current optimization path and starts over in a different region of the likelihood function which results in much higher chances of finding the global maximum. But this robustness against multimodality of the objective function comes at the cost of much higher computational demands.

As the EM algorithm is computationally highly demanding, even in its basic form, and tends to become tediously slow when close to convergence our estimation routine relies on a hybrid estimation algorithm (Render and Walker, 1984): It first uses either the EM or the SAEM algorithm and takes advantage of their robustness before it switches to the direct 
maximization of the log likelihood by the much faster BFGS algorithm. The estimation routine in this form turned out to be efficient and robust as it reliably converged towards the same maximum likelihood estimates regardless of the randomly chosen start values. 
Working Papers of the Socioeconomic Institute at the University of Zurich

The Working Papers of the Socioeconomic Institute can be downloaded from http://www.soi.uzh.ch/research/wp/index2.html

$0708 \quad$ Rationality on the Rise: Why Relative Risk Aversion Increases with Stake Size, Helga Fehr-Duda, Adrian Bruhin, Thomas F. Epper, and Renate Schubert, July 2007, 30p.

$0707 \quad$ I'm not fat, just too short for my weight - Family Child Care and Obesity in Germany, Philippe Mahler, May 2007, 27p.

0706 Does Globalization Create Superstars?, Hans Gersbach and Armin Schmutzler , April 2007, 23 p.

0705 Risk and Rationality: Uncovering Heterogeneity in Probability Distortion, Adrian Bruhin, Helga Fehr-Duda, and Thomas F. Epper, July 2007 (revised version), 29p.

$0704 \quad$ Count Data Models with Unobserved Heterogeneity: An Empirical Likelihood Approach, Stefan Boes, March 2007, 26p.

0703 Risk and Rationality: The Effect of Incidental Mood on Probability Weighting, Helga Fehr, Thomas Epper, Adrian Bruhin, and Renate Schubert, February 2007, 27p.

0702 Happiness Functions with Preference Interdependence and Heterogeneity: The Case of Altruism within the Family, Adrian Bruhin and Rainer Winkelmann, February 2007, 20p.

0701 On the Geographic and Cultural Determinants of Bankruptcy, Stefan Buehler, Christian Kaiser, and Franz Jaeger, June 2007 (revised version), 35 p.

0610 A Product-Market Theory of Industry-Specific Training, Hans Gersbach and Armin Schmutzler, November 2006, 28 p.

0609 Entry in liberalized railway markets: The German experience, Rafael Lalive and Armin Schmutzler, April 2007, revised version, 20 p.

0608 The Effects of Competition in Investment Games, Dario Sacco and Armin Schmutzler, April 2007, revised version, 22 p.

0607 Merger Negotiations and Ex-Post Regret, Dennis Gärtner and Armin Schmutzler, September 2006, 28 p.

$0606 \quad$ Foreign Direct Investment and R\&D offshoring, Hans Gersbach and Armin Schmutzler, June 2006, $34 \mathrm{p}$.

0605 The Effect of Income on Positive and Negative Subjective Well-Being, Stefan Boes and Rainer Winkelmann, May 2006, 23p.

$0604 \quad$ Correlated Risks: A Conflict of Interest Between Insurers and Consumers and Its Resolution, Patrick Eugster and Peter Zweifel, April 2006, 23p.

0603 The Apple Falls Increasingly Far: Parent-Child Correlation in Schooling and the Growth of Post-Secondary Education in Switzerland, Sandra Hanslin and Rainer Winkelmann, March 2006, 24p.

0602 Efficient Electricity Portfolios for Switzerland and the United States, Boris Krey and Peter Zweifel, February 2006, 25p.

$0601 \quad$ Ain't no puzzle anymore: Comparative statics and experimental economics, Armin Schmutzler, February 2006, 45 p.

0514 Money Illusion Under Test, Stefan Boes, Markus Lipp and Rainer Winkelmann, November 2005, 7p.

0513 Cost Sharing in Health Insurance: An Instrument for Risk Selection? Karolin Becker and Peter Zweifel, November 2005, 45p.

0512 Single Motherhood and (Un)Equal EducationalOpportunities: Evidence for Germany, Philippe Mahler and Rainer Winkelmann, September 2005, 23p.

0511 Exploring the Effects of Competition for Railway Markets, Rafael Lalive and Armin Schmutzler, April 2007, revised version, 33p. 
0510 The Impact of Aging on Future Healthcare Expenditure; Lukas Steinmann, Harry Telser, and Peter Zweifel, September 2005, 23p.

0509 The Purpose and Limits of Social Health Insurance; Peter Zweifel, September 2005, $28 p$.

0508 Switching Costs, Firm Size, and Market Structure; Simon Loertscher and Yves Schneider, August 2005, 29p.

0507 Ordered Response Models; Stefan Boes and Rainer Winkelmann, March 2005, 21p.

0506 Merge or Fail? The Determinants of Mergers and Bankruptcies in Switzerland, 19952000; Stefan Buehler, Christian Kaiser, Franz Jaeger, March 2005, 18p.

0505 Consumer Resistance Against Regulation: The Case of Health Care Peter Zweifel, Harry Telser, and Stephan Vaterlaus, February 2005, 23p.

0504 A Structural Model of Demand for Apprentices Samuel Mühlemann, Jürg Schweri, Rainer Winkelmann and Stefan C. Wolter, February 2005, 25p.

0503 What can happiness research tell us about altruism? Evidence from the German Socio-Economic Panel Johannes Schwarze and Rainer Winkelmann, February 2005, 26p.

0502 Spatial Effects in Willingness-to-Pay: The Case of Nuclear Risks Peter Zweifel, Yves Schneider and Christian Wyss, January 2005, 37p.

$0501 \quad$ On the Role of Access Charges Under Network Competition Stefan Buehler and Armin Schmutzler, January 2005, 30p.

0416 Social Sanctions in Interethnic Relations: The Benefit of Punishing your Friends Christian Stoff, Dezember 2004, 51p.

0415 Single Motherhood and (Un)equal Educational Opportunities: Evidence from Germany, Philippe Mahler and Rainer Winkelmann, November 2004, 23p.

$0414 \quad$ Are There Waves in Merger Activity After All? Dennis Gärtner and Daniel Halbheer, September 2004, 39p.

0413 Endogenizing Private Information: Incentive Contracts under Learning By Doing Dennis Gärtner, September 2004, 32p.

0412 Validity and Reliability of Willingness-to-pay Estimates: Evidence from Two Overlapping Discrete-Choice Experiments Harry Telser, Karolin Becker and Peter Zweifel. September 2004, 25p.

0411 Willingness-to-pay Against Dementia: Effects of Altruism Between Patients and Their Spouse Caregivers, Markus König und Peter Zweifel, September 2004, 22p.

$0410 \quad$ Age and Choice in Health Insurance: Evidence from Switzerland Karolin Becker and Peter Zweifel, August 2004, 30p.

$0409 \quad$ Vertical Integration and Downstream Investment in Oligopoly Stefan Buehler and Armin Schmutzler, July 2004, 30p.

$0408 \quad$ Mergers under Asymmetric Information - Is there a Lemons Problem? Thomas Borek, Stefan Buehler and Armin Schmutzler, July 2004, 38p.

0407 Income and Happiness: New Results from Generalized Threshold and Sequential Models, Stefan Boes and Rainer Winkelmann, June 2004, 30p.

$0406 \quad$ Optimal Insurance Contracts without the Non-Negativity Constraint on Indemnities Revisited, Michael Breuer, April 2004, 17p.

0405 Competition and Exit: Evidence from Switzerland Stefan Buehler, Christian Kaiser and Franz Jaeger, March 2004, 28p.

$0404 \quad$ Empirical Likelihood in Count Data Models: The Case of Endogenous Regressors Stefan Boes, March 2004, 22p. 